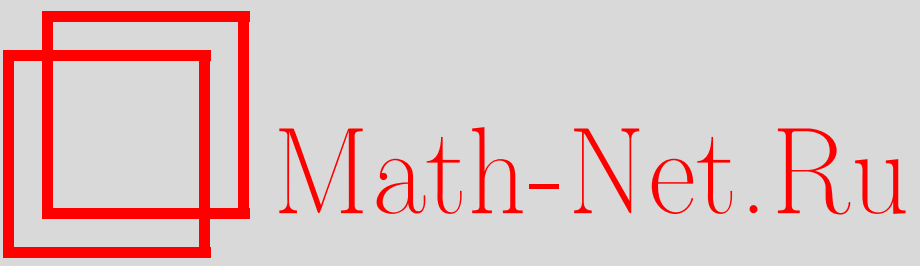

Д. Ю. Волченков, М. Ю. Налимов, Поправки на сжимаемость жидкости к спектрам развитой турбулентности, ТМФ, 1996, том 106, номер 3, 375-389

DOI: https://doi.org/10.4213/tmf1122

Использование Общероссийского математического портала Math-Net.Ru подразумевает, что вы прочитали и согласны с пользовательским соглашением

http://www.mathnet.ru/rus/agreement

Параметры загрузки:

IP: 18.208 .226 .222

26 апреля 2023 г., 15:35:56 


\title{
ПОПРАВКИ НА СЖИМАЕМОСТЬ ЖИДКОСТИ К СПЕКТРАМ РАЗВИТОЙ ТУРБУЛЕНТНОСТИ
}

\begin{abstract}
Построено регулярное разложение для случая слабой сжимаемости в теории развитой турбулентности изотропной однородной сжимаемой жидкости с функционалом действия MSR-типа. Параметром разложения является число Маха-Ма. В инерционном интервале теории несжимаемой жидкости изучены инфракрасные сингулярности, определяемые поперечными полями, характерными для теории несжимаемой жидкости. Данные особенности связаны с составными операторами поперечных полей, которые были исследованы методами квантово-полевой ренормгруппы. В результате было показано, что поперечные поля индуцируют скейлинговое поведение в теории, при этом скейлинговая размерность числа Ма равна $1 / 3$ (т.е. Ма $k^{-1 / 3}$ есть скейлинговобезразмерный параметр корреляционных функций теории в инерционном интервале).
\end{abstract}

\section{1. ВВЕДЕНИЕ}

Метод квантово-полевой ренормгруппы (РГ) успешно применялся для описания спектральных свойств в инерционном интервале развитой турбулентности несжимаемой жидкости, например, в работах [1-3]. В них используется стохастическое уравнение Навье-Стокса

$$
\partial_{t} \mathbf{v}=\frac{1}{\rho}\left(\eta \nabla^{2} \mathbf{v}+\eta^{\prime} \partial(\partial \mathbf{v})-\partial p\right)-(\mathbf{v} \partial) \mathbf{v}+\mathbf{f},
$$

где $\mathbf{v}(\mathbf{x}, t)$ - векторное поле скорости, $\rho(\mathbf{x}, t)$ - поле плотности жидкости, $\eta$ - сдвиговая вязкость, $\eta^{\prime}=(\eta / 3+\zeta), \zeta$ - объемная вязкость, $p(\mathbf{x}, t)$ - поле давления, $\mathbf{f}(\mathbf{x}, t)$ - плотность случайной внешней силы, для которой предполагается гауссово распределение с коррелятором

$$
\left\langle f_{i}(\mathbf{x}, t) f_{j}\left(\mathbf{x}^{\prime}, t^{\prime}\right)\right\rangle=D_{i j}\left(x-x^{\prime}\right) \delta\left(t-t^{\prime}\right) .
$$

Скобки $\langle\cdots\rangle$ означают здесь усреднение по случайной силе, а функция $D_{i j}$ моделирует подкачку энергии в систему из крупномасштабной области.

Для несжимаемой жидкости уравнение неразрывности

$$
\partial_{t} \rho+\partial_{i}\left(\rho v_{i}\right)=0
$$

диктует поперечность поля $\mathbf{v}$. Сжимаемая жидкость описывается той же системой (1), $(2),(3)$, которую следует дополнить уравнением состояния $p=p(\rho)$. Считая сжимаемость жидкости малой, вместо уравнения состояния можно использовать уравнение 
адиабатической связи отклонений $\delta p$ и $\delta \rho$ от равновесных значений $p_{0}, \rho_{0}$ :

$$
\delta p=c^{2} \delta \rho
$$

$c$ - скорость звука (в дальнейшем мы будем опускать символ $\delta$ при этих отклонениях и считать равновесное значение плотности $\left.\rho_{0}=1\right)$. В теории появляется новый безразмерный параметр - число Маха: Ма $=v_{c} / c$, где $v_{c}$ - характерная скорость турбулентных пульсаций.

Обобшение на случай сжимаемаемой жидкости феноменологической теории Колмогорова (приведенное, например, в [4]) вводит в инерционном интервале новую переменную, пропорциональную Ма $/ k^{1 / 3}$, где $k$ - волновое число. Аналогичный результат получен в [5] в рамках уравнений самосогласования. Интересным представляется проверка данного результата в последовательном статистическом подходе. В работе [6] декларировано полное решение этой проблемы при произвольных числах Ма непосредственным применением метода РГ в формулировке Вильсона. Однако мы считаем данную работу недостоверной. Используя формально более развитый, хотя и эквивалентный, квантово-полевой РГ-подход, в приложении к данной статье мы объясняем, что рассматриваемая теория (1)-(4) неренормируема и к ней нельзя прямо применять РГподход (в [6] вопрос ренормируемости не обсуждается вовсе, авторы ограничились однопетлевой ренормировкой одной из функций Грина).

Поэтому в [4] была рассмотрена теория (1)-(4) в приближении слабой сжимаемости жидкости, т.е. исследована первая поправка к спектрам в инерционном интервале несжимаемой жидкости по малому числу Ма. Из уравнений (1)-(3) с точностью до первого порядка по формально малому параметру $c^{-2}$ поля продольной компоненты скорости и давления были выражены в локальном приближении по времени как составные операторы случайных полей поперечных пульсаций. После изучения ренормировки этих операторов был подтвержден результат [5] и определено, что первая поправка к спектрам турбулентности несжимаемой жидкости пропорциональна $\mathrm{Ma}^{2} / k^{2 / 3}$. Таким образом, результаты $[4,5]$ свидетельствуют, что эти поправки формально существенны в инфракрасной (ИК) области спектра, а значит, $c^{-2}$ является массивным параметром в скейлинговой области. В теории турбулентности несжимаемой жидкости нижняя граница инерционного интервала определяется ИК-массой (обратным интегральным масштабом турбулентности), которая содержится в модельной функции распределения случайной силы. Новый, связанный со сжимаемостью, массивный параметр начиная с некоторых чисел Ма попадает в инерционный интервал. При достаточно большом Ма вообше не останется интервала волновых чисел, в котором возможно построение теории турбулентности, не содержашей размерных параметров. Поэтому с точки зрения теории скейлингового поведения основная задача будет заключаться не в установлении размерностей, а в описании так называемых скейлинговых функций.

В некотором смысле первый шаг к решению этой задачи сделан в данной статье. Здесь предложен метод построения разложения турбулентных спектров по числу Ма (нулевой член разложения соответствует несжимаемой жидкости). Сущность предлагаемого метода заключается в следующем. Предъявлено квантово-полевое действие теории, в котором при вершинах взаимодействия новых полей (отсутствующих в теории турбулентности несжимаемой жидкости) стоят предполагаемые малыми множители, пропорциональные $c^{-2}$. В этой теории предлагается сперва проводить усреднение по поперечным полям и затем воспользоваться результатами РГ для несжимаемой жидкости. Тогда в вершины взаимодействия новых полей войдут составные операторы поперечных полей с известными скейлинговыми размерностями. Эти операторы и 
определяют ИК-поведение в теории турбулентности сжимаемой жидкости со скейлинговыми размерностями полей и параметров : $\Delta[c]=-1 / 3, \Delta[p]=-2 / 3, \Delta\left[v_{\|}\right]=-1$ $\left(v_{\|}\right.$- продольная компонента скорости). Скейлинговая размерность времени оказалась равной $\Delta_{t}=-2 / 3$, как и без учета сжимаемости [2]. Показано, что ИК-сушественные вклады поправок на сжимаемость жидкости представляются в виде рядов по переменной $c^{-2} k^{-2 / 3}$ (первый порядок разложения совпадает с определенным в [4]). В теории с учетом сжимаемости появляется новая соответствуюшая времени универсальная переменная $c k t$.

Показано, что в ведущем порядке по $c^{-2} k^{-2 / 3}$ в конкретных диаграммах теории возмушений пропагаторы новых полей, связанных со сжимаемостью, дают $\delta(t)$-образный вклад в поперечные функции Грина, что оправдьвает использованное в [4] $t$-локальное приближение.

\section{2. ТЕОРИЯ ВОЗМУЩЕНИЙ В СЖИМАЕМОЙ ЖИДКОСТИ}

Стохастическая задача (1)-(4), дополненная условиями запаздывания и начальными условиями нулевых флуктуаций полей при $t \rightarrow \infty$ эквивалентна квантово-полевой задаче с функционалом действия MSR-типа [7]:

$$
\begin{gathered}
S=\frac{1}{2} v_{i}^{\prime} D_{i j} v_{j}^{\prime}+v_{i}^{\prime}\left[-\partial_{t} v_{i}-v_{j} \partial_{j} v_{i}+\frac{1}{1+p / c^{2}}\left(\eta \nabla^{2} v_{i}+\eta^{\prime} \partial_{i} \partial_{j} v_{j}-\partial_{i} p\right)\right]+ \\
+p^{\prime}\left(-\frac{1}{c^{2}} \partial_{t} p-\partial_{i} v_{i}-\frac{1}{c^{2}} v_{i} \partial_{i} p-\frac{1}{c^{2}} p \partial_{i} v_{i}\right) .
\end{gathered}
$$

Необходимые интегрирования по $\mathbf{x}, t$ и суммирования по повторяющимся индексам полей здесь и в аналогичных формулах в дальнейшем подразумеваются. Производящий функционал корреляционных функций теории (1)-(4) определяется формулой $G\left(A_{\Phi}\right)=$ $\int D v^{\prime} D v D p D p^{\prime} \operatorname{det} M \exp \left(S+\Phi A_{\Phi}\right)$, где $\Phi=\left\{v, v^{\prime}, p, p^{\prime}\right\}, A_{\Phi}$ - источники полей, а $\operatorname{det} M$ при определенных ограничениях на диаграммную технику, обсуждаемых в [2], представляет собой несущественный множитель. При $c^{-2}=0$ интегрирование по полю $p^{\prime}$ в производяшем функционале с действием (5) приводит к появлению $\delta\left(\partial_{i} v_{i}\right)$, поэтому поле скорости $\mathbf{v}$ становится поперечным, и мы получаем теорию несжимаемой жидкости. Действие последней $S_{i c}$ получается из (5) при условии $p^{\prime}=p=0$ и замене $\mathbf{v}^{\prime}$ и $\mathbf{v}$ на поперечные (в импульсном представлении) поля. Поэтому параметр $c^{-2}$ определяет поправки на сжимаемость жидкости. Наша задача заключается в построении теории возмушений по $1 / c$. Введем обозначения для продольных и поперечных компонент векторных полей $\mathbf{v}$ и $\mathbf{v}^{\prime}: \mathbf{v}_{\perp}=P_{\perp} \mathbf{v} ; \mathbf{v}_{\|}=P_{\|} \mathbf{v} ; \quad P_{\|_{i j}}=k_{i} k_{j} / k^{2} ; P_{\perp i j}=\delta_{i j}-P_{\|_{i j}}$ (аналогично для $v_{\perp}^{\prime}$ и $v_{\|}^{\prime}$ ).

При $c^{-2}=0$ в теории (5) отсутствуют продольные поля, уравнение (3) дает $v_{\|} \sim$ $c^{-2}$. Поэтому естественной представляется замена переменных $v_{\|} \rightarrow \tilde{u}, p^{\prime} \rightarrow q^{\prime}$ в действии (5):

$$
v_{\|}=\tilde{u} / c^{2} ; \quad p^{\prime}=c^{2} q^{\prime}
$$

Квантово-полевая теория возмушений для производяшего функционала $G(A)$ получается в результате разложения $\exp S$ по вершинам (нелинейностям), т.е. членам, содержашим произведения трех и более полей. Для построения соответствующей диаграммной техники следует представить $1 /\left(1+p / c^{2}\right)$ в виде ряда по $p / c^{2}$. Чтобы эта 
диаграммная техника естественно согласовьвалась с разложением по $c^{-1}$, полезно добиться того, чтобы вершины взаимодействия, содержащие продольные и скалярные поля $v_{\|}, v^{\prime} \|, p$ и $p^{\prime}$ (т.е. те поля, которые отсутствуют в теории турбулентности несжимаемой жидкости), были пропорциональны отрицательным степеням $c$. Для этого сделаем в теории с действием (5) следуюшую замену переменных $p \rightarrow q, \tilde{u} \rightarrow u$ :

$$
p=q-F_{p} \equiv \tilde{q} ; \quad \tilde{u}=u+F_{v 1}-F_{v 2} q,
$$

где $F_{p}=\Delta^{-1} \partial_{i} \partial_{k} v_{\perp i} v_{\perp k}, F_{v 1}=\partial_{i} \Delta^{-1} \mathcal{D}_{t} \Delta^{-1} \partial_{j} \partial_{k} v_{\perp j} v_{\perp k}$ и $F_{v 2}=\Delta^{-1} \partial_{i} \mathcal{D}_{t}, \mathcal{D}_{t}=$ $\partial t+\left(v_{\perp} \partial\right)$. Очевидно, что связанный с этим преобразованием якобиан тривиален. Замена переменных (7) включает в себя предложенные в [4] сдвиги полей $p$ и $v_{\|}$, поэтому нами будут учтены рассмотренные в [4] поправки к спектрам несжимаемой турбулентности.

В результате замен (6)-(7) действие (5) принимает вид

$$
\begin{aligned}
& S(\Phi)=\frac{1}{2} v_{i \perp}^{\prime} D_{\perp i j} v_{\perp j}^{\prime}+\frac{1}{2} v_{\|_{i}}^{\prime} D_{\|_{i j}} v_{\|_{j}}^{\prime}+v_{\perp j}^{\prime}\left[-\partial_{t} v_{\perp i}-v_{\perp j} \partial_{j} v_{\perp i}-\right. \\
& \left.-\frac{1}{c^{2}} v_{\perp j} \partial_{j} \tilde{u}_{i}-\frac{1}{c^{2}} \tilde{u}_{j} \partial_{j} v_{\perp i}+\frac{1}{1+\tilde{q} / c^{2}} \eta \Delta v_{\perp i}-\frac{1}{c^{4}} \frac{\tilde{q}}{1+\tilde{q} / c^{2}}\left(\eta+\eta^{\prime}\right) \Delta \tilde{u}_{i}\right]+ \\
& +v_{\|_{i}}^{\prime}\left[-\frac{1}{c^{2}} \partial_{t} \tilde{u}_{i}-\frac{1}{c^{2}}\left(v_{\perp j} \partial_{j} \tilde{u}_{i}+\tilde{u}_{j} \partial_{j} v_{\perp i}\right)-\frac{1}{c^{4}} \tilde{u}_{j} \partial_{j} \tilde{u}_{i}-\frac{1}{c^{2}} \frac{\tilde{q}}{1+\tilde{q} / c^{2}} \eta \Delta v_{\perp i}+\right. \\
& \left.\quad+\frac{1}{c^{2}} \frac{1}{1+\tilde{q} / c^{2}}\left(\eta+\eta^{\prime}\right) \Delta \tilde{u}_{\| i}-\partial_{i} q+\frac{1}{c^{2}} \frac{\tilde{q}}{1+\tilde{q} / c^{2}} \partial_{i} \tilde{q}\right]-p^{\prime}\left[\partial_{i} u_{i}+\frac{1}{c^{2}} \partial_{i}\left(\tilde{u}_{i} \tilde{q}\right)\right] .
\end{aligned}
$$

В действии (8) мы различаем корреляторы $D_{\perp}$ и $D_{\|}$поперечных и продольных компонент случайной силы. Чтобы продольная часть уравнения (1) не противоречила утверждению $v_{\|} \sim 1 / c^{2}$, необходимо предположить $f_{\|} \sim 1 / c^{2}$, откуда следует $D_{\|} \sim 1 / c^{4}$. Поэтому по аналогии с $[1,2]$ выберем корреляторы в виде

$$
D_{\perp i j}(k)=P_{\perp i j} g_{0} \nu_{0}^{3} k^{4-d-2 \varepsilon}, \quad D_{\|_{i j}}(k)=\frac{1}{c^{4}} P_{\|_{i j}} g_{0}^{\prime} k^{4-d-2 \varepsilon},
$$

где $k$ - импульс, $\nu_{0}=\eta / \rho_{0}$ - кинематическая вязкость, $d$ - размерность пространства. Множитель $\nu_{0}{ }^{3}$ введен в выражение для $D_{\perp}(9)$ для обезразмеривания константы $g_{0}$. Физическим значением параметра регулярного разложения РГ-функций в теории турбулентности несжимаемой жидкости является $\varepsilon=2[1,2]$. Мы будем рассматривать случай трехмерного пространства $d=3$.

Для краткости в дальнейшем обозначим $v_{\perp}=v, v_{\perp}^{\prime}=v^{\prime}$. Существенным является то, что действие (8) обладает той же галилеевой инвариантностью, что и теория турбулентности несжимаемой жидкости [2], а именно, оно инвариантно относительно преобразований полей $\mathbf{v}(t, \mathbf{x}) \rightarrow \mathbf{v}(t, \mathbf{x}+\mathbf{a} t)-\mathbf{a}, \Phi(t, \mathbf{x}) \rightarrow \Phi(t, \mathbf{x}+\mathbf{a} t)$, где $\Phi-$ остальные поля, кроме $\mathbf{v}$. Чтобы в этом убедиться, необходимо учесть, что продольное поле $\tilde{u}$ или $u$ представимо в виде градиента некоторого скалярного потенциала, поэтому $\partial_{i} \tilde{u}_{j}=\partial_{j} \tilde{u}_{i}$. Это свойство было использовано нами при написании действия (8) (оно позволило отбросить член $v_{i}^{\prime} \tilde{u}_{j} \partial_{j} \tilde{u}_{i}$, поскольку $\tilde{u}_{j} \partial_{j} \tilde{u}_{i}$ - чисто продольный оператор), и с его помощью можно написать $v_{i}^{\prime} v_{j} \partial_{j} \tilde{u}_{i}=-v_{i}^{\prime} \tilde{u}_{j} \partial_{i} v_{j}$. После чего поперечное поле $\mathbf{v}$ входит в действие (8) либо под знаком производной, либо в ковариантной производной $\mathcal{D}_{t}$. 


\section{3. ЭФФЕКТИВНАЯ ТЕОРИЯ В ИК-ОБЛАСТИ}

Действие (8) состоит из действия теории несжимаемой жидкости $S_{i c}$ и членов, содержаших продольные и скалярные поля $u, u^{\prime}, q, p^{\prime}$. Для $S_{i c}$ ИК-поведение в инерционном интервале установлено методом РГ в работах $[1,2]$, где показано, что скейлинговые размерности полей и их аргументов равны $\Delta_{v}=-1 / 3, \Delta_{v}^{\prime}=10 / 3, \Delta_{t}=-2 / 3$ при физических значениях параметров $\varepsilon, d$ (как обычно, размерность импульса $d_{k}=1$ ). Мы воспользуемся результатами этих работ для суммирования ИК-особенностей в подграфах, состоящих только из поперечных линий в обычной диаграммной технике в теории (8).

ТАБЛИША 1

Поперечные операторы теории сжимаемой жидкости

\begin{tabular}{|r|c|c|c|c|}
\hline № & Оператор & Размерность & Поля & Фактор \\
\hline 1 & $v^{\prime} \partial v$ & 4 & $u$ & $1 / c^{2}$ \\
\hline 2 & $\Delta F_{v 1} F_{p}{ }^{l}$ & $1-2 l / 3$ & $u^{\prime}, q^{n}$ & $1 / c^{2(l+n+1)}$ \\
\hline 3 & $v^{\prime} \Delta F_{p}{ }^{l}$ & $3 \frac{2}{3}-2 l / 3+\omega$ & $q^{n}$ & $1 / c^{2(l+n)}$ \\
\hline 4 & $\Delta F_{v 2} F_{p}{ }^{l}$ & $1 \frac{2}{3}-2 l / 3$ & $u^{\prime}, q^{n+1}$ & $1 / c^{2(l+n+1)}$ \\
\hline 5 & $v^{\prime} \Delta F_{v_{1}} F_{p}{ }^{l}$ & $4 \frac{1}{3}-2 l / 3$ & $q^{n}$ & $1 / c^{2(l+n+1)}$ \\
\hline 6 & $\partial F_{p}{ }^{l+1}$ & $1-2(l+1) / 3$ & $u^{\prime}, q^{n}$ & $1 / c^{2(l+n)}$ \\
\hline 7 & $v^{\prime} \Delta F_{p}{ }^{l},(l+n)>0$ & $5 \frac{1}{3}-2 l / 3$ & $u, q^{n}$ & $1 / c^{2(l+n+1)}$ \\
\hline 8 & $\partial F_{v 1}$ & 0 & $p^{\prime}, q$ & $1 / c^{2}$ \\
\hline 9 & $v^{\prime} \Delta F_{v_{2}} F_{p}{ }^{l}(l+n)>0$ & $5-2 l / 3$ & $q^{n+1}$ & $1 / c^{2(l+n+1)}$ \\
\hline 10 & $\partial F_{v 2}$ & $2 / 3$ & $p^{\prime}, q^{2}$ & $1 / c^{2}$ \\
\hline 11 & $\mathcal{D}_{t} F_{v 1}$ & $-1 / 3$ & $u^{\prime}$ & $1 / c^{2}$ \\
\hline 12 & $\partial F_{p}$ & $1 / 3$ & $u, p^{\prime}$ & $1 / c^{2}$ \\
\hline 13 & $F_{v 1} \partial v$ & $-1 / 3$ & $u^{\prime}$ & $1 / c^{2}$ \\
\hline 14 & $F_{v 1} \partial F_{p}$ & $-2 / 3$ & $p^{\prime}$ & $1 / c^{2}$ \\
\hline 15 & $\mathcal{D}_{t} F_{v 2}$ & $1 / 3$ & $q, u^{\prime}$ & $1 / c^{2}$ \\
\hline 16 & $F_{v 2} \partial F_{p}$ & 0 & $q, p^{\prime}$ & $1 / c^{2}$ \\
\hline 17 & $F_{v 2} \partial v$ & $1 / 3$ & $q, u^{\prime}$ & $1 / c^{2}$ \\
\hline 18 & $\partial F_{v 1}$ & 0 & $u, u^{\prime}$ & $1 / c^{4}$ \\
\hline 19 & $\partial v$ & $2 / 3$ & $u, u^{\prime}$ & $1 / c^{2}$ \\
\hline 20 & $\partial F_{v 2}$ & $2 / 3$ & $q, u, u^{\prime}$ & $1 / c^{4}$ \\
\hline 21 & $F_{v 2} \partial F_{v 1}$ & -1 & $u^{\prime}$ & $1 / c^{4}$ \\
\hline 22 & $\Delta F_{p}{ }^{l}$ & $2-2 l / 3$ & $u, q^{n}, u^{\prime}$ & $1 / c^{2(n+l+1)}$ \\
\hline 23 & $F_{v 1} \partial F_{v 2}$ & $-1 / 3$ & $q, u^{\prime}$ & $1 / c^{4}$ \\
\hline 24 & $v^{\prime} \partial F_{v 2}$ & $3 \frac{2}{3}$ & $q$ & $1 / c^{2}$ \\
\hline 25 & $F_{v 2} \partial F_{v 2}$ & $1 / 3$ & $u^{\prime}, q^{2}$ & $1 / c^{4}$ \\
\hline 26 & $v^{\prime} v \partial F_{v 1}$ & 3 & - & $1 / c^{2}$ \\
\hline 27 & $\Delta v F_{p}{ }^{l}, \quad(l+n)>0$ & $12 / 3-2 l / 3$ & $u^{\prime}, q^{n}$ & $1 / c^{2(l+n)}$ \\
\hline
\end{tabular}

Тогда в производящем функционале корреляционных функций сперва выполняется усреднение по поперечным полям $v, v^{\prime}$, а уже потом строится разложение по вершинам, 
содержащим продольные и скалярные поля. Соответствующая диаграммная техника будет состоять из линий полей $u^{\prime}, u, q, p^{\prime}$ и “одетых" пропагаторов и вершин поперечных полей $v, v^{\prime}$. Пропагаторы продольных и скалярных полей присоединяются к своим собственным вершинам или к составным операторам поперечных полей.

Скейлинговые размерности составных операторов поперечных полей, входящих в действие (8), известны нам из работ $[4,8]$ и вычисляются по аналогии с [4]. Эти размерности приведены в таблице 1 .

Во второй графе таблицы схематично (без указания индексной структуры и с произвольным расположением производных) представлены составные операторы поперечных полей, к которым в действии (8) присоединяются продольные и скалярные поля, указанные в графе 4. Степень $c^{-2}$ при соответствуюшей вершине взаимодействия приведена в графе 5 . Множители $1 /\left(1+\tilde{q} / c^{2}\right)$ в действии (8) мы рассматриваем в виде разложения по $\tilde{q} / c^{2}$, поэтому в некоторых строках таблицы приведены сведения о размерностях бесконечных последовательностей операторов при любых $l, n \geq 0$ (иногда указаны дополнительные ограничения на $l, n$, обусловленные индексной структурой полей, т.е. продольностью и поперечностью). Третий столбец таблицы дает скейлинговую размерность соответствующего составного оператора при физических значениях $d=3, \varepsilon=2$. ИК-поправочный индекс теории $\omega$ в старших порядках теории возмушений не известен, однако в соответствии с результатами первого порядка $\varepsilon$-разложения и с общей идеологией $\omega>0$ (его положительность необходима для существования ИК-устойчивой фиксированной точки уравнения РГ теории развитой турбулентности в несжимаемой жидкости).

Скейлинговые размерности составных операторов с произвольным числом вставок операторов $F_{p}$ ранее были неизвестны. Мы их определили методом, аналогичным использованному в [4]. Обозначим простой линией пропагатор $\Delta_{v v}$, линией со стрелкой $-\Delta_{v v^{\prime}}$ (стрелка направлена в сторону поля $v^{\prime}$ ), волнистой линией - оператор $\Delta^{-1}$, а штрихом - пространственную производную. В таких обозначениях, например, первый неисследованый в [4] составной оператор из семейства 3 представляется рис. 1.

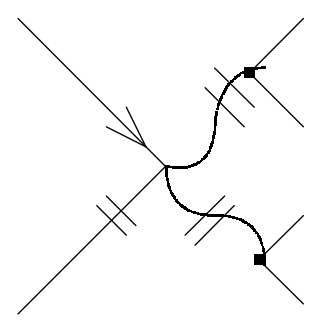

Рис. 1

Известно, что при ренормировке к нелокальному оператору могут примешиваться локальные той же канонической размерности. Каноническая размерность оператора в логарифмической теории определяется как сумма канонических размерностей входяших в оператор полей и производных $\left(\operatorname{dim} v=\operatorname{dim} \partial=1, \operatorname{dim} v^{\prime}=\operatorname{dim} \partial_{t}=2\right.$ при $d=3$ ), поэтому $\operatorname{dim} F_{p}=2, \operatorname{dim} F_{v 1}=3, \operatorname{dim} F_{v 2}=1$, откуда следует, что каноническая размерность рассматриваемого оператора равна 9 . Этот оператор, как и все операторы, представленные в табл. 1, галилеево-инвариантный, поэтому к нему могут примешиваться при ренормировке только галилеево-инвариантные локальные состав- 
ные операторы. Как и в [4], имеются веские основания полагать, что эти локальные операторы ИК-несущественны по сравнению с нелокальными. Поэтому ограничимся рассмотрением элементов матрицы ренормировки, ответственных за смешивание нелокальных операторов.

Для операторов, рассмотреннных в [4], скейлинговая размерность определялась как сумма размерностей входящих в них локальных фрагментов, производных и $\Delta^{-1}$ операторов. При ренормировке оператора, представленного на рис. 1 , такой же результат получается после устранения расходимостей подграфов, помещенных в штриховые рамки на рис. 2 (в результате диагональный элемент матрицы ренормировки данного составного оператора равен произведению констант ренормировки оператора $v^{\prime} \Delta v$ и двух операторов $v v)$.

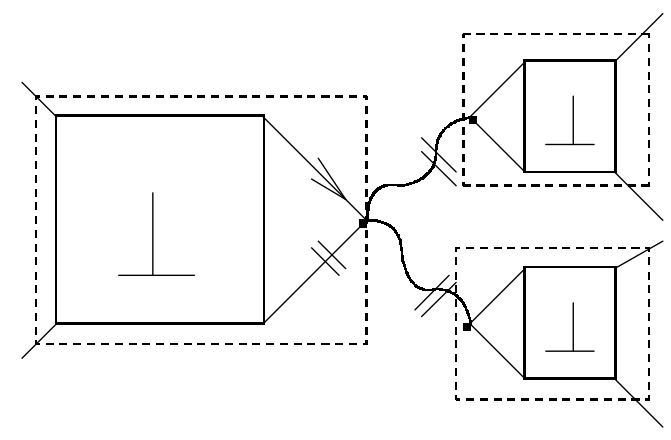

Рис. 2

Но в отличие от [4] к данному оператору при ренормировке могли бы примешиваться и нелокальные составные операторы типа $v_{i}^{\prime} \Delta v_{i} \Delta^{-2}\left(\partial_{j} v_{l}\right)\left(\partial_{l} v_{m}\right)\left(\partial_{m} v_{n}\right)\left(\partial_{n} v_{j}\right)$ из-за расходимости подграфа, помешенного в штриховую рамку на рис. 3 .

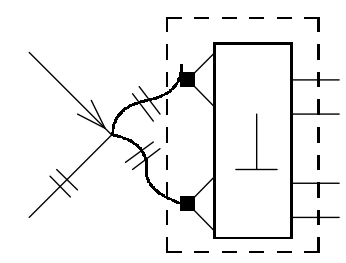

Рис. 3

Однако на самом деле этот оператор не примешивается к рассматриваемому благодаря все тому же свойству галилеевой инвариантности. Действительно, мысленно стянув в точку подграф, обведенный штриховой линией на рис. 3 , мы получаем в качестве локального фрагмента оператор $\partial_{j} \partial_{l} \partial_{m} \partial_{n}\left(v_{l} v_{m} v_{n} v_{j}\right)$, который галилеевонеинвариантен (хотя включает в себя галилеево-инвариантный $\left(\partial_{j} v_{l}\right)\left(\partial_{l} v_{m}\right)\left(\partial_{m} v_{n}\right) \times$ $\left.\left(\partial_{n} v_{j}\right)\right)$, а потому не примешивается к данному.

Таким образом, даже в классе нелокальных составных операторов в принципе возможны нетривиальные смешивания при ренормировке за счет примеси новых локальных фрагментов к одному или нескольким исходным. Из приведенных рассуждений сле- 
дует, что для обсуждения этой проблемы требуется рассмотреть ренормировку корреляционных функций с несколькими вставками локальных составных операторов. Более подробно этот вопрос освещен в приложении 2.

Для конкретного оператора, представленного на рис. 1, следует, что по соображениям галилеевой инвариантности к паре фрагментов $\partial_{i} \partial_{j} v_{i} v_{j}, \partial_{l} \partial_{m} v_{l} v_{m}$ при ренормировке могут примешиваться лиш галилеево-инвариантные операторы типа $\Delta^{2} \partial_{i} \partial_{j} v_{i} v_{j}$, а к паре $v_{i}^{\prime} \Delta v_{i}, \partial_{l} \partial_{m} v_{l} v_{m}$ - типа $\partial_{l} \partial_{m}\left(\partial_{j} v_{i}^{\prime} \partial_{i} v_{j} \partial_{l} v_{m}\right)$. Логично предположить, что примешивающиеся операторы менее ИК-сушественны, поскольку содержат большое количество производных, каждая из которых повышает размерность оператора на единицу. Таким образом, наиболее ИК-сушественный вклад в рассматриваемый оператор, видимо, имеет скейлинговую размерность, равную сумме скейлинговых размерностей $v^{\prime} \Delta v$, двух $\partial_{l} \partial_{m} v_{l} v_{m}$ и двух $\Delta^{-1}$.

Скейлинговые размерности всех операторов в табл. 1 были определены с учетом того, что $\Delta\left[v^{\prime} \Delta v\right]=11 / 3+\omega, \Delta\left[F_{p}\right]=-2 / 3, \Delta\left[F_{v 1}\right]=-1, \Delta\left[F_{v 2}\right]=-1 / 3, \Delta\left[v^{\prime} v\right]=3$ (cм. $[4,9])$.

Мы намерены подтвердить результаты [4] о скейлинговой размерности $\Delta[c]=-1 / 3$. При таком предположении все операторы, получаемые разложением $\left(1+\tilde{q} / c^{2}\right)^{-1}$ в ряд по $F_{p} / c^{2}$, одинаково ИК-существенны. Ниже будет определена скейлинговая размерность поля $q: \Delta[q]=-2 / 3$, поэтому также одинаково сушественны все члены разложения этих множителей по $q / c^{2}$. Тогда операторы табл. 1 можно сравнивать между собой по любым представителям указанных под определенными номерами семейств.

В сооответствии со своими скейлинговыми размерностями семейства операторов № 5 и № 9 менее ИК-существенны, чем № 3 . Оператор из семейства № 3 с $n=1$ несуществен по сравнению с № 24. Поэтому семейства операторов № 3,5 и 9 могут в дальнейшем не рассматриваться. Оператор из № 7 при $n=0$ несушествен по сравнению с № 1 ; № 27 и № 2 при $n=0$ несушественны по сравнению с № 11, а № 4 - по сравнению с № 15 , оператор № 22 при $n=0$ и $l=1$ - по сравнению с № 18. В духе идеологии Ландау следует отбросить все указанные ИК-несушественные операторы для получения эффективного действия, описываюшего ИК-асимптотики рассматриваемой теории.

Однако это действие мы приведем несколько позже, т.к. существует возможность его еше упростить. После замен переменных (6), (7) квадратичная часть действия (8) приобретает вид

$$
\begin{gathered}
S_{2}=\frac{1}{2} v_{i}^{\prime} D_{\perp i j} v_{j}^{\prime}+\frac{1}{2} u_{i}^{\prime} D_{\|_{i j}} u_{j}^{\prime}+v_{i}^{\prime}\left(-\partial_{t} v_{i}+\nu \Delta v_{i}\right)+u_{i}^{\prime}\left(-\frac{1}{c^{2}} \partial_{t} u_{i}+\frac{1}{c^{2}} \nu^{\prime} \Delta u_{i}-\right. \\
\left.-\partial_{i} q-\frac{1}{c^{2}} \nu^{\prime} \partial i \partial_{t} q+\frac{1}{c^{2}} \Delta^{-1} \partial_{i} \partial^{2}{ }_{t} q\right)-p^{\prime} \partial_{i} u_{i}
\end{gathered}
$$

где $\nu$ - кинематическая вязкость, $\nu=\eta / \rho_{0}$ (напомним, что $\left.\rho_{0}=1\right)$, а $\nu^{\prime}=\left(\eta+\eta^{\prime}\right) / \rho_{0}$. Выражение (10) определяет пропагаторы теории

$$
\begin{aligned}
\Delta_{v^{\prime} v} & =\frac{P_{\perp}}{-i \omega+\nu k^{2}}, \quad \Delta_{v v}=\frac{D_{\perp} P_{\perp}}{\omega^{2}+\nu^{2} k^{4}} \\
\Delta_{q q} & =\frac{k^{2} D_{\|} c^{4}}{\left(\omega^{2}-k^{2} c^{2}\right)^{2}+\nu^{\prime 2} \omega^{2} k^{4}}, \quad \Delta_{p^{\prime} q}=\frac{-i \omega-\nu^{\prime} k^{2}}{\omega^{2}-c^{2} k^{2}-i \nu^{\prime} \omega k^{2}} \\
\Delta_{p^{\prime} u} & =\frac{i k_{i}}{k^{2}}, \quad \Delta_{u^{\prime} q}=-\frac{c^{2} i k_{i}}{\omega^{2}-c^{2} k^{2}-i \nu^{\prime} \omega k^{2}} .
\end{aligned}
$$


Остальные пропагаторы равны нулю. При переходе в импульсно-частотное представление в пропагаторах продольных и скалярных полей интегралы по $\omega$ вычисляются по вычетам в полюсах $\omega \approx-i \nu^{\prime} k^{2} / 2 \pm k c$, где в вешественной части значения полюса мы пренебрегли членами порядка $\nu^{\prime 2} k^{2} / c^{2}$ (малыми как за счет малых $k$ в ИК-области, так и ввиду больших $c$ ). В результате пропагаторы (11) можно записать в виде

$$
\begin{aligned}
\Delta_{v^{\prime} v} & =P_{\perp} \theta(t) e^{-\nu k^{2} t}, \quad \Delta_{v v}=P_{\perp} \frac{D_{\perp}}{2 \nu k^{2}} e^{-\nu k^{2}|t|}, \\
\Delta_{q q} & \cong c^{2} D_{\|} e^{-\nu^{\prime} k^{2}|t| / 2} \cos (c k|t|) / 2 k^{2} \nu^{\prime}, \quad \Delta_{p^{\prime} q} \cong-e^{-\nu^{\prime} k^{2} t / 2} \theta(t) \cos (c k t), \\
\Delta_{p^{\prime} u} & \cong i k_{i} \delta(t) / k^{2}, \quad \Delta_{u^{\prime} q} \cong-c \theta(t) k_{i} e^{-\nu^{\prime} k^{2} t / 2} \sin (c k t) / k .
\end{aligned}
$$

При физических значениях $\varepsilon, d$ скейлинговая размерность времени, вычисляемая в "поперечной науке", равна $-2 / 3$. Поэтому безразмерная переменная $c k t$ в пропагаторах (12) также определяет размерность $c$ как $\Delta[c]=-1 / 3$. Тогда по пропагаторам (12) можно определить скейлинговые размерности продольных и скалярных полей:

$$
\Delta[q]=-\frac{2}{3}, \quad \Delta\left[p^{\prime}\right]=3 \frac{2}{3}, \quad \Delta[u]=-1, \quad \Delta\left[u^{\prime}\right]=3 \frac{1}{3} .
$$

Таким образом, все члены в $\tilde{q}$ и $\tilde{u}$ оказались одних размерностей (одинаково сушественны). В соответствии с (13) дальнейшее сокрашение табл. 1 не представляется возможным (все оставшиеся составные операторы одинаково сушественны), зато можно утверждать ИК-несушественность вершин взаимодействия $\nu^{\prime} u^{\prime} \Delta u q^{l} / c^{2 l}$ действия (8) для любого $l$. В результате, оставляя лиш ИК-сушественные члены (8), эффективное действие теории развитой турбулентности сжимаемой жидкости можно написать в виде

$$
\begin{aligned}
S(\Phi)=\frac{1}{2} v_{i}^{\prime} D_{\perp i j} v_{j}^{\prime}+\frac{1}{2} u_{i}^{\prime} D_{\|_{i j}} u_{j}^{\prime}+ & \\
& +v_{i}^{\prime}\left(-\partial_{t} v_{i}-v_{j} \partial_{j} v_{i}-\frac{1}{c^{2}} v_{j} \partial_{j} \tilde{u}_{i}-\frac{1}{c^{2}} \tilde{u}_{j} \partial_{j} v_{i}+\nu \Delta v_{i}\right)+ \\
& +u_{i}^{\prime}\left(-\frac{1}{c^{2}} \partial_{t} \tilde{u}_{i}+\frac{1}{c^{2}} \nu^{\prime} \Delta u_{i}-\frac{1}{c^{2}}\left(v_{j} \partial_{j} \tilde{u}_{i}+\tilde{u}_{j} \partial_{j} v_{i}\right)-\right. \\
& \left.-\frac{1}{c^{2}} \nu^{\prime} \partial_{i} \partial_{t} q+\frac{1}{c^{2}} \Delta^{-1} \partial_{i} \partial_{t}^{2} q-\frac{1}{c^{4}} \tilde{u}_{j} \partial_{j} \tilde{u}_{i}-\partial_{i} q+\frac{1}{c^{2}} \frac{\tilde{q}}{\left(1+\frac{\tilde{q}}{c^{2}}\right)} \partial_{i} \tilde{q}\right)+ \\
& +p^{\prime}\left(-\partial_{i} u_{i}-\frac{1}{c^{2}} \tilde{u}_{i} \partial_{i} \tilde{q}-\frac{1}{c^{2}} \tilde{q} \partial_{i} \tilde{u}_{i}\right) .
\end{aligned}
$$

В действии (14) следует провести ренормировку поперечных полей и их составных операторов. Оно естественным образом представляется в виде суммы $S_{i c}+S_{c}$, где $S_{i c}$ - действие теории несжимаемой жидкости [2], $S_{c}$ - добавка к нему за счет учета сжимаемости. Все вершины взаимодействия из $S_{c}$ содержат малости $c^{-2}, c^{-4}$.

\section{4. ОСОБЕННОСТИ ПРЕДЛАГАЕМОЙ ТЕОРИИ ВОЗМУЩЕНИЙ ПО $c^{-2}$}

В [4] утверждается, что поправки первого порядка по $c^{-2}$ к корреляционным функциям поперечных полей определяются вставкой составного оператора $c^{-2} v^{\prime} v \partial F_{v 1}$ (№ 26 
в табл. 1). Чтобы подтвердить это, необходимо уточнить степени по $c$ вкладов в корреляционные функции вершин и пропагаторов (14).

Некоторые вершины взаимодействия (14) содержат производные по времени. Входящая в операторы $F_{v 2}$ производная $\partial_{t}$, действуя на $\sin c k t$ и $\cos c k t$ в пропагаторах (12), дает лишний множитель $c$. Следует также отметить, что в случае больших $c$ зависимость от времени в пропагаторах продольных и скалярных полей складывается из быс-

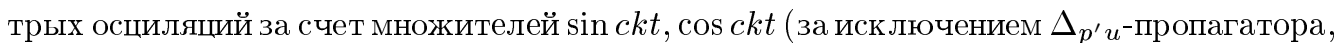
вклад которого в диаграммную технику с точки зрения зависимости от $t$ очевиден и в дальнейшем обсуждаться не будет) на фоне медленного затухания, обеспечиваемого множителями $\exp \left\{-\nu^{\prime} k^{2} t / 2\right\}$.

В функции Грина, составленные из пропагаторов только поперечных полей, время входит в виде инвариантной переменной $\bar{\nu} k^{2} t$, где $\bar{\nu}-$ РГ-инвариант, на который следует заменить $\nu$ после выполнения интегрирования $\int \mathcal{D} v^{\prime} \mathcal{D} v$ (т.е. после вычисления диаграмм, составленных из поперечных линий, которое осуществляется в теории возмушений по $g$ ). Известно [2], что $\bar{\nu} \sim k^{-4 / 3}$, поэтому времени в теории турбулентности несжимаемой жидкости и приписывается скейлинговая размерность $\Delta[t]=-2 / 3$. В продольных и скалярных пропагаторах (12) скейлингово-безразмерная переменная - $k c t$, а вторая связанная со временем переменная $\nu^{\prime} k^{2} t$ является ИК-несушественной в скейлинговой области, поскольку $\nu^{\prime}$ не заменяется на $\bar{\nu}^{\prime}$. Однако содержащие данную комбинацию множители должны быть оставлены в пропагаторах (12), чтобы обеспечить затухание по времени, и могут быть опушены лиш в интегралах, которые сходятся сами по себе. Последнее бывает, если продольная или скалярная линия присоединена обоими концами к подблоку поперечных линий (где имеет место затухание $\left.\exp \left\{-\bar{\nu} k^{2} t\right\}\right)$.

Для определения порядка теории по $c^{-1}$, в который дает вклад та или иная диаграмма, полезно утверждение, что в ведушем порядке по $c^{-1}$ в пропагаторах продольных и скалярных линий (12) ("одетых", т.е. с включением подграфов без поперечных линий) $c \sin c k t \sim \delta(k t)$ (доказывается в полной аналогии с известным представлением $\delta$-функции $\left.\delta(t)=-1 / 2 \lim _{N} \rightarrow \infty N e^{-N|t|}\right)$. Аналогично для линий, присоединенных обоими концами к поперечному подблоку, можно утверждать, что $c^{2} \cos c k t \sim \bar{\nu} \delta(t)$, $\partial_{t} \cos c k t \sim \delta(t)$. Учитывая $D_{\|} \sim 1 / c^{4}$, для таких линий справедливо

$$
\begin{aligned}
& \Delta_{q q} \sim c^{-4}, \quad \Delta_{p^{\prime} q} \sim c^{-2}, \quad \Delta_{p^{\prime} u} \sim 1, \quad \Delta_{u^{\prime} q} \sim 1, \\
& \partial_{t} \Delta_{q q} \sim c^{-2}, \quad \partial_{t} \Delta_{p^{\prime} q} \sim 1, \quad \partial_{t}^{2} \Delta_{q q} \sim 1, \quad \partial_{t} \Delta_{u^{\prime} q} \sim 1 .
\end{aligned}
$$

Поэтому в отношении поправок на сжимаемость в корреляторы поперечных полей диаграммы со вставками продольных и скалярных линий оказываются старшего порядка по $c^{-2}$ по сравнению с результатами [4]. Кроме отрицательных степеней $c$ в вершинах (14) дополнительную малость по $c^{-1}$ содержат пропагаторы $\Delta_{q q}, \Delta_{p^{\prime} q}$ и $\partial_{t} \Delta_{q q}$. Из (14), (15) можно утверждать, что в каждый порядок по $c^{-2}$ дает вклад конечное число диаграмм. Это касается не только поправок на сжимаемость к корреляторам поперечных полей, но и к описываемым предлагаемой теорией корреляционным функциям с внешними продольными и скалярными полями.

В заключение отметим еше одну особенность, присушую предлагаемой диаграммной технике. Вершины взаимодействия (14) содержат до двух производных по времени. Пропагаторы $\Delta_{u^{\prime} q}, \Delta_{p^{\prime} q}$ и $\Delta_{v^{\prime} v}$ пропорциональны $\theta(t)$, а $\Delta_{q q}$ и $\Delta_{v v}$ зависят от $|t|$, поэтому, например, $\partial_{t} \Delta_{p^{\prime} q}$ и $\partial_{t}^{2} \Delta_{q q}$ содержат члены, пропорциональные $\delta(t)$. Это приводит к появлению неопределенностей типа $\theta(0)$ в диаграммах, содержащих подграфы вида, представленного на рис. 4 , и сингулярностей, пропорциональных $\delta(0)$, в диа- 


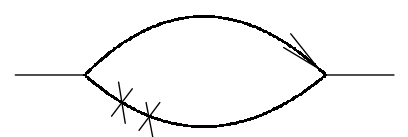

Рис. 4

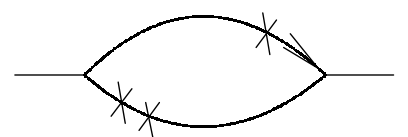

Рис. 5

граммах, содержаших подграфы вида, представленного на рис. 5. На обоих рисунках крестиком на линии отмечена производная по времени.

Аналогичная проблема обсуждалась в [4] (составной оператор, который рассматривался в этой работе также содержал $\partial_{t}$ ), но в нашем случае ситуация принципиально иная. Дело в том, что в отличие от [4] мы не изменили исходных стохастических уравнений (1)-(4), поэтому можем сравнивать результаты нашей диаграммной техники с таковыми, получаемыми непосредственным итерированием уравнений (1)-(4) с последующим усреднением по случайной силе. При этой итерационной процедуре не возникает обсуждаемых сингулярностей. В нашей же диаграммной технике они появились в результате замен функциональных переменных интегрирования (7) (с тривиальным якобианом). Поскольку результаты двух теорий возмушений должны совпадать, вышеуказанные неопределенности и сингулярности в предлагаемой диаграммной технике сокращаются сами по себе. Достаточно предъявить подходящую регуляризацию. В качестве таковой мы предлагаем заменить $\delta(t)$ в корреляторах случайных сил (12) на $\lim _{N} \rightarrow \infty(N / \sqrt{\pi}) \exp \left\{-N^{2} t^{2} / 2\right\}$ и дополнить диаграммную технику предписанием перекидывать производные $\partial_{t}$ в вершинах так, чтобы на линии со штрихованным полем (за исключением $\Delta_{p^{\prime}}$ ) действовало не более одной производной $\partial_{t}$. Тогда сингулярности находят свое выражение в положительных степенях $N$ и можно проследить их сокрашение.

Работа выполнена при финансовой поддержке Российского фонда фундаментальных исследований (проект 93-02-14515) и Международного научного фонда (ISF grant R$63000)$.

ПРИЛОЖКЕНИЕ 1

Ренормируемость теории (1)-(4) удобнее обсуждать, рассматривая действие MSRтипа, записанное в полях $v, \rho, v^{\prime}, \rho^{\prime}$ :

$$
\begin{aligned}
& S=\frac{1}{2} v_{i}^{\prime} D_{i j} v_{j}^{\prime}+v_{i}^{\prime}\left(-\partial_{t} v_{i}-v_{j} \partial_{j} v_{i}+\frac{1}{1+\rho}\left(\eta \nabla^{2} v_{i}+\eta^{\prime} \partial_{i} \partial_{j} v_{j}-c^{2} \partial_{i} \rho\right)\right)+ \\
& \quad+\rho^{\prime}\left(-\partial_{t} \rho-\partial_{i}\left(v_{i} \rho\right)\right)
\end{aligned}
$$

(как обычно, $\rho^{\prime}$ и $v^{\prime}$ - вспомогательные поля). Учитывая (9) и полагая, как обычно, импульсную размерность $d^{p}$ и частотную размерность $d^{\omega}$ равными единице, а также определив полную размерность как $d=d^{p}+2 d^{\omega}[2]$, получим канонические размерности полей и переменных действия (табл. 2). 
ТАБЛИЦА 2

Канонические размерности полей и переменных

\begin{tabular}{|l|c|c|c|c|c|cc|c|}
\hline$d /$ Поля и параметры & $v^{\prime}$ & $v$ & $\rho^{\prime}$ & $\rho$ & $c^{2}$ & $\eta, \eta^{\prime}$ & $g$ \\
\hline$d^{p}$ & $d+1$ & -1 & $d$ & 0 & -2 & -2 & $2 \varepsilon$ \\
\hline$d^{\omega}$ & -1 & 1 & 0 & 0 & 2 & 1 & 0 \\
\hline$d$ & $d-1$ & 1 & $d$ & 0 & 2 & 0 & $2 \varepsilon$ \\
\hline
\end{tabular}

Регулярное $\varepsilon$-разложение строится от логарифмической точки $\varepsilon=0$, в которой константа $g$ безразмерна. В соответствии со своей канонической размерностью параметр $c^{2}$ УФ-несушественньй, поэтому ренормировку теории можно изучать, отбросив члены действия (16), при которых стоит коэффициентом $c^{2}$ (аналогично переходу к безмассовой теории при ренормировке теории $\varphi^{4}$ ), рассматривая отброшенные члены в качестве составных операторов. Пропагаторы теории (16) получаются из (11) заменами $p \rightarrow c^{2} \rho$, $p^{\prime} \rightarrow \rho^{\prime} c^{2}$ и $v_{\|} \rightarrow c^{2} v_{\|}-c^{2} \Delta^{-1} \partial_{i} \partial_{t} \rho($ с учетом $(6)-(7))$ и нетривиально зависят от $c^{2}:$

$$
\begin{aligned}
& \Delta_{v_{\|} v_{\|}}=\frac{\omega^{2} c^{4} D_{\|}}{\left(\omega^{2}-k^{2} c^{2}\right)^{2}+\nu^{\prime 2} \omega^{2} k^{4}}, \quad \Delta_{\rho^{\prime} \rho}=\frac{-i \omega-\nu^{\prime} k^{2}}{\omega^{2}-c^{2} k^{2}-i \nu^{\prime} \omega k^{2}}, \\
& \Delta_{\rho^{\prime} v_{\|}}=-\frac{i k_{i} c^{2}}{\omega^{2}-c^{2} k^{2}-i \omega \nu^{\prime} k^{2}}, \quad \Delta_{v_{\|}^{\prime} \rho}=-\frac{i k_{i}}{\omega^{2}-c^{2} k^{2}-i \omega \nu^{\prime} k^{2}}, \\
& \Delta_{v_{\|}^{\prime} v_{\|}}=-\frac{i \omega}{\omega^{2}-k^{2} c^{2}-i \nu^{\prime} k^{2}}, \quad \Delta_{\rho \rho}=\frac{k^{2} D_{\|}}{\left(\omega^{2}-k^{2} c^{2}\right)^{2}+\nu^{\prime 2} \omega^{2} k^{4}} .
\end{aligned}
$$

Несложно убедиться, что теория (15) с отброшенным пропорциональным $c^{2}$ членом нетривиальна (хотя в ней и отсутствуют пропагаторы $\Delta_{v_{\|} \rho^{\prime}}$ и $\Delta_{v_{\|} v_{\|}}$; здесь мы не будем полагать $\left.D_{\|} \sim 1 / c^{4}\right)$.

При $\varepsilon=0$ теория тогда не содержит размерных параметров, поверхностные расходимости в ней определяются каноническими размерностями полей (табл. 2). Несложный анализ показывает, что поверхностно расходятся 1-неприводимые диаграммы с внешними линиями $\left\{v v^{\prime}\right\},\left\{v^{\prime} \rho\right\},\left\{\rho \rho^{\prime}\right\},\left\{v v v^{\prime}\right\},\left\{v v v v^{\prime}\right\}$, а также вышеперечисленные с добавлением произвольного числа внешних линий $\rho$-типа, поскольку полная каноническая размерность поля $\rho$ равна нулю. Каждому типу расходящихся 1-неприводимых диаграмм соответствует новый тип контрчленов в действии ренормированной теории (мы не включили в список расходяшихся функций Грина формально расходяшиеся функции типа $\left\langle v^{\prime} v^{\prime}\right\rangle$, т.к. контрчлены должны быть локальны по импульсам и частотам, и учли, что 1-неприводимые функции без внешних штрихованных полей обрашаются в ноль, как и в случае несжимаемой жидкости [2], а также что структура взаимодействия (15) обеспечивает вынесение производной на внешнюю линию $\rho^{\prime}$-типа в любой диаграмме, что устраняет некоторые расходимости).

Таким образом, для ренормировки теории (15) требуется бесконечное число контрчленов, причем все эти члены маргинальны (в них не входят размерные параметры). Следовательно, эта теория неренормируема, попытка применения метода ренормгруппы к ней наталкивается на необходимость поиска фиксированной точки в бесконечной системе $\beta$-функций зарядов и исследования ее устойчивости. Прецеденты решения этой проблемы нам неизвестны. 
ПРИЛОЖЕНИЕ 2

В теории критического поведения традиционно делается неконтролируемое предположение: при построении эффективного действия (гамильтониана) из гамильтониана Гинзбурга-Ланадау, который обычно имеет вид бесконечного ряда, отбрасываются все члены, являющиеся ИК-несушественными по своей канонической размерности в логарифмической теории. Оправданием этого шага обычно признается то, что при рассмотрении отброшеннных членов в качестве составных операторов их скейлинговые размерности вычисляются в виде суммы канонической размерности и поправок по параметру регулярного разложения $\varepsilon$, которые при малых $\varepsilon$ не превышают разности канонических размерностей. Поскольку данное рассуждение касается всевозможных локальных составных операторов, а при ренормировке функций Грина с несколькими вставками составных операторов к ним примешиваются лиш составные операторы большей канонической размерности, обычно достаточно ограничиться изучением одной вставки каждого составного оператора. Конечно, интересуюшей нас физической теории соответствует достаточно большое значение параметра $\varepsilon$, размерности при физическом $\varepsilon$ (во всех порядках разложения) всех составных операторов нам никогда неизвестны, поэтому возможность перехода к конечному эффективному действию от полного гамильтониана Гинзбурга-Ландау всегда является лишь гипотезой, оправдываемой полученными результатами.

Особенность теории развитой турбулентности заключается в том, что вследствие галилеевой инвариантности нам точно известны скейлинговые размерности полей, времени и некоторых составных операторов во всех порядках по $\varepsilon$ (при физическом значении $\varepsilon=2, d=3[2,4,9]: \Delta[v]=-1 / 3, \Delta\left[v^{\prime}\right]=10 / 3, \Delta[t]=-2 / 3, \Delta\left[\partial_{i} v_{j} \partial_{i} v_{j}\right]=0$, $\Delta\left[v^{\prime} \Delta v\right]=3+\omega, \Delta\left[v^{n}\right]=-n / 3$ для любого $\left.n\right)$. Этими результатами мы воспользовались при построении эффективного действия (14).

При изучении скейлинговых размерностей нелокальных операторов (табл. 1 из раздела 2) нам впервые реально пришлось рассматривать ренормировку функций Грина с более чем одной вставкой составного оператора. Хотя в этой ренормировке нет ничего принципиально нового, насколько нам известно, этот вопрос ранее не обсуждался в литературе. Поэтому в данном приложении мы рассмотрим его более подробно.
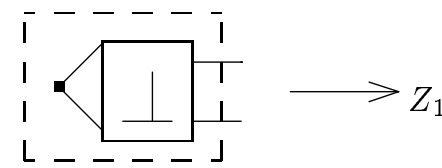

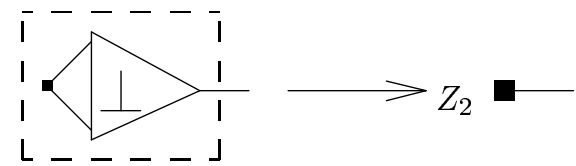

Рис. 6

Обозначим некоторый составной оператор (например, $v^{2}$ ) точкой с двумя выходящими линиями. Ренормировка составного оператора (в функциях Грина с одной вставкой) осуществляется со смешиванием, если поверхностно расходятся 1-неприводимые диаграммы с этим оператором и разным числом внешних линий (или коэффициент при расходимости диаграммы имеет сложную структуру значков или производных). Например, в случае поверхностной расходимости обведенных подграфов на рис. 6 следует заключить, что рассматриваемый оператор ренормируется сам (для простоты мы ограничились случаем, когда у поля $v$ отсутствует константа ренормировки $Z_{v}$, как это 
имеет место в теории турбулентности несжимаемой жидкости) и что к нему примешивается оператор, линейный по полю $v$.

Известно, что поверхностные расходимости диаграмм локальны, т.е. представляются в виде некоторой константы $Z$, умноженной на локальный оператор (см. рис. 6). Константы $Z$ безразмерны, поэтому примешиваюшиеся операторы должны иметь те же канонические размерности, что и исходный оператор $F$ (например, $\partial v$ для $v^{2}$ ).

Рассмотрим ренормировку функций Грина со вставками двух составных операторов $F$ (см. рис. 7 ). В соответствии со стандартным размерным анализом поверхностно расходиться при этом могут новые типы 1-неприводимых диаграмм (что для случая оператора $v^{2}$ означает примесь операторов $v^{4}, v^{2} \partial v, \partial v \partial v, v \partial^{2} v$ и $\left.\partial^{3} v\right)$.
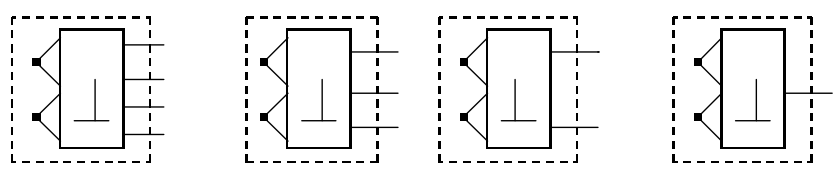

Рис. 7

При увеличении числа вставок оператора $F$ семейство примешивающихся операторов еще увеличивается. Можно утверждать, что к нескольким операторам $F$ могут примешиваться все локальные составные операторы, каноническая размерность которых равна сумме канонических размерностей рассматриваемого набора операторов $F$.

Этот факт учитывался нами при определении скейлинговых размерностей операторов табл. 1 (раздел 2) и при получении эффективного действия (14). Однако для строгого обоснования наших результатов (касаюшихся ренормировки бесконечного числа вставок составных операторов) требуется знание скейлинговых размерностей всех локальных операторов, которым мы не обладаем. Поэтому мы пользовались следующими правдоподобными соображениями.

Обсуждая ИК-существеннность операторов при физическом значении $\varepsilon$, мы вместо канонической размерности оператора используем сумму скейлинговых размерностей входящих в него полей и производных. Это справедливо для операторов типа $v^{n}$, которые, имея отрицательную скейлинговую размерность, крайне ИК-существенны. Однако они галлилеево-неинвариантны и поэтому не примешиваются к рассматриваемым нами.

Вспоминая [2], что логарифмическую теорию можно выбрать при некоторой размерности пространства $d \neq 3$, заключаем, что при смешивании операторов постоянным является число полей $v^{\prime}$ (его каноническая размерность зависит от $d$ ), поэтому при построении примешиваюшегося оператора в отличие от случая $d=3$ нельзя заменить два поля $v^{\prime}$ (оцениваемых по размерности как 20/3) на галлилеево-инвариантную комбинацию $\partial v \partial v$ (оценка размерности для нее дает $4 / 3$, и потому она более ИК-сушественна).

В рассматриваемых операторах накапливается комбинация $\partial_{i} v_{k} \partial_{k} v_{i}$, скейлинговая размерность которой известна точно (4/3). Но при ренормировке к $n$ операторам $\partial_{i} v_{k} \partial_{k} v_{i}$ не примешивается оператор $(\partial v)^{n}$ (с разными расстановками значков), поскольку из каждого $\partial_{i} v_{k} \partial_{k} v_{i}$ производные выносятся наружу из 1-неприводимой диаграммы, а операторы $\partial \partial \cdots \partial(v v \cdots v)$ не являются галиллево-инвариантными. Поэтому к $n$ операторам $(\partial v)^{2}$ может примешаться разве что $\partial^{n}(\partial v)^{n / 2}$, в котором слишком много производных, так что его логично считать несушественным. 
$\mathrm{K}$ паре операторов $v^{\prime} \Delta v$ (признанных нами несушественными из-за сдвижки их размерности на $\omega$ ) может примешиваться $\partial v^{\prime} \partial v \partial v^{\prime} \partial v$, размерность которого нам неизвестна. Поэтому строго обосновать допустимость использования идеологии Ландау для получения эффективного действия (14) мы не можем, впрочем как всегда в теории критического поведения.

\section{Список литературы}

[1] De Dominicis C. Martin P. C. // Phys Rev A. 1979. V. 29. P. 419.

[2] Аджемян Л.Ц., Васильев А.Н., Письмак Ю.М. // ТМФ. 1983. Т. 57. №2. С. 268.

[3] Аджемян Л.Ц., Антонов Н.В., Васильев А.Н. // ЖЭТФ. 1989. Т. 95. Вып. 4. С. 1272.

[4] Аджемян Л.ЦІ, Налимов М. Ю., Степанова М. М. // ТМФ. 1995. Т. 104. № 2. С. 260270.

[5] Львов В.С., Михайлов А.В. Препринт № 54. Новосибирск: ИАиЭ, 1977.

[6] Staroselsky I., Yakhot V., Kida S., Orszag A. S. // Phys Rev. Lett. 1990. V. 65. № 2. P. 171.

[7] Martin P. C., Siggia E. D., Rose H. A. // Phys Rev. A. 1973. V. 8. P. 423.

[8] Аджемян Л. Ц., Ким Т. Л. // Вестн. С.-П. ун-та, сер 4: Физика, химия. 1992. Вып. 3. (№ 18) C. 71.

[9] Аджемян Л.Ц., Васильев А.Н., Гнатич М. // ТМФ. 1988. Т. 74. № 2. С. 180.

Санкт-Петербургский

государственный университет
Поступила в редакцию 27.III.1995 г.

\section{Yu. Volchenckov, M. Yu. Nalimov \\ THE CORRECTIONS TO FULLY DEVELOPED TURBULENT SPECTRA DUE TO THE COMPRESSIBILITY OF FLUID}

We construct the regular expansion at small compressibility for the theory of fully developed turbulence of isotropic homogeneous compressible fluid with the action of MSR-type. The parameter of the expansion is the Mach number Ma. In the inertial range (of compressible fluid) the infrared peculiarities determined by the transversal fields (used in the theory of incompressible fluid) are studied. These peculiarities are connected with the composite operators of transversal fields, which are investigated by the quantum-field renormalization group method. As a result, it is shown that the transversal fields induce the scaling behavior in the theory proposed with the scaling dimension of $\mathrm{Ma}$ equal to $1 / 3$ (i.e., $\mathrm{Ma} k^{-1 / 3}$ is the scaling dimensionless parameter of the correlation functions in the inertial range). 